\title{
Profile and Performance of Rigid Gas Permeable and Scleral Lenses on Keratoconic Patients in the Developing Contact Lens Practice Settings
}

\author{
Walter Kibet Yego ${ }^{1 *}$, Harun Chemjor ${ }^{2}$ \\ ${ }^{1}$ Department of Optometry and Vision Sciences, Masinde Muliro University of Science and Technology, Kakamega, Kenya \\ ${ }^{2}$ Department of Health Professional Education, Masinde Muliro University of Science and Technology, Kakamega, Kenya \\ Email: *drkibetwalter@gmail.com
}

How to cite this paper: Yego, W.K. and Chemjor, H. (2020) Profile and Performance of Rigid Gas Permeable and Scleral Lenses on Keratoconic Patients in the Developing Contact Lens Practice Settings. Open Journal of Ophthalmology, 10, 191-199.

https://doi.org/10.4236/ojoph.2020.103021

Received: May 3, 2020

Accepted: June 27, 2020

Published: June 30, 2020

Copyright $\odot 2020$ by author(s) and Scientific Research Publishing Inc. This work is licensed under the Creative Commons Attribution International License (CC BY 4.0).

http://creativecommons.org/licenses/by/4.0/

\begin{abstract}
Purpose: To present the profile of keratoconic contact lens wearers, alongside with performance of corneal and scleral rigid gas permeable (RGP) at different stages of keratoconus based on limited diagnostic resources. Methods: 5-year Clinical records of keratoconic corneal and scleral RGP contact lens wearers were retrieved. Data on age, gender, visual acuity (VA), refraction (RE), stage of keratoconus and mode of correction among other variables were obtained for analysis. Information pertaining to the outcome in pre and post-fit at different severity was determined. Results: A total of 124 medical records were analyzed, with the age $(20.86 \pm 9.50$ years), gender ( $58.9 \%$ male and $41.1 \%$ female), Laterality ( $57.3 \%$ bilateral, $26.6 \%$ right eye and left eye as $16.1 \%$ ) and ethnicity (91.1\% Africans and $8.9 \%$ Asians). There were no significant differences in effects of lenses (RGP and Scleral lenses) across three stages in visual acuity and in three stages of severity $\mathrm{H}_{\mathrm{RGP}}(2)=1.05, p=0.59 ; \mathrm{H}_{\text {scleral }}(1)=2.24, p=0.134$. Similar non-significant effect was observed in refractive error correction $\mathrm{H}_{\mathrm{RGP}}$ $(2)=1.62, p=0.44: \mathrm{H}_{\text {scleral }}(1)=1.143, p=0.285$. Conclusion: The profile of $\mathrm{KC}$ contact lens wearer was comparable to other studies in developing setting. Keratoconic grading should be based on available resources. Corneal rigid gas permeable and scleral lenses were beneficial to keratoconic patients in respect of refractive error correction and visual improvement to keratoconic patient.
\end{abstract}

\section{Keywords}

Keratoconus, Visual Outcome, Rigid Gas Permeable, Scleral Lenses, Developing Practices 


\section{Introduction}

Keratoconus (KC), as a non-inflammatory corneal ectasia, causes severe visual impairment if management is delayed, thus leading to dire visual burden among affected patients especially in low-income settings [1] [2] [3]. Patients suffer from symptoms of distortion in vision and irregular cornea (detected during retinoscopy and topographical mapping) that worsens with time. In basic examination settings, slit lamp signs may include Vogt's striae, Fleischer's ring as well as scarring of the cornea [4].

Keratoconus progressive nature calls for timely management using both optical and surgical treatments options. Surgically, collagen cross-linking (CXL), intra-stromal ring segment implants at an early stage and corneal transplantation in the late stages are recommended [5] [6]. In developing settings, optical corrections provide better options of management due to fewer complications and availability of cheaper options [1]. Spectacles, which remain the most affordable in low-income settings, cannot adequately correct for the irregular astigmatism caused by steep protruding cones. Therefore, the need arises to consider more effective options such as soft contact lenses, hybrid and rigid gas permeable (RGP) contact lenses which remain costly. Corneal and scleral contact lenses remain the most preferred options for KC management due to their excellent vision outcome as compared to spectacles [7]. Moreover, if fitted timely and skillfully, corneal and scleral contact lenses may provide avenues to negate and even postpone the need for corneal transplant [8] [9].

Despite the efforts in timely management, early detection is limited by the inadequate possession of diagnostic equipment especially in the resources constrained settings [10]. In such instances, practitioners own basic slit lamps and keratometers as equipment of $\mathrm{KC}$ diagnosis and management. Visual outcome varies depending on the methods of grading (which guides on intervention) and management options themselves which are thought to play a key role in patients' quality of life and productivity. Until recently, visual outcome expectations in keratoconus in developing clinical settings have been extrapolated based on findings of other studies despite the clinical setting possessing basic equipment such as keratometer and slit lamp alone [9] [10] [11]. In East Africa, most specialist contact lens clinics still use basic equipment for diagnosis and cheaper contact lenses for management of KC. Therefore, there is need to present realistic expected results based on diagnostic and grading methods available as well as visual outcome in two lens modalities in the practices. Several studies in Africa have concentrated on the profile of general KC patients [11] [12] [13] [14] [15] and none on the contact wearers. The aim of the present study is to present the profile and the performance of corneal and scleral lenses on patients' eyes as well as patients' demographic characteristics.

\section{Methods}

The study employed a retrospective design of 5-year keratoconic patients' medi- 
cal records at Muthaiga Eye Clinic in Nairobi, Kenya. These records constituted consented patients who were examined and fitted with corneal and scleral rigid gas permeable (RGP) contact lenses between March 2014 and 2019. Ethical approval was obtained from the Masinde Muliro University of Science and Technology Ethical and Review Committee (MMUST IERC). The study adhered to the tenets of Helsinki Declaration. Records of patients with history of corneal surgery (including penetrating keratoplasty), incomplete records on demographic and visual findings or active ocular pathologies were excluded from the analysis.

Information on patients' age, gender, presenting complaints, associated conditions, ethnicity involved eyes (unilateral or bilateral); refraction (final spherical equivalents) and visual acuity (before and after the correction either with corneal RGP or scleral contact lenses and keratometric (K-readings) readings using manual keratometer were extracted. In addition, contact lens parameters (where applicable), Lens dioptric power, and Total diameter (TD) of RGP and scleral lenses to determine contact lens parameters used in keratoconus correction were recorded. Patients' grading was limited K-values obtained by manual keratometers owing to the lack of the corneal topographer. Therefore, the Collaborative Longitudinal Evaluation of Keratoconus (CLEK) method was preferred for classifying the severity of KC based on steepest keratometric reading [16].

$\begin{array}{ll}\text { Mild } & \mathrm{K}_{\text {steeper }}<45 \text { diopters }(\mathrm{D}) \\ \text { Moderate } & 45<\mathrm{K}_{\text {steeper }}<52 \mathrm{D} \\ \text { Severe } & \mathrm{K}_{\text {steeper }}>52 \mathrm{D}\end{array}$

The refraction (in spherical equivalent in the case of astigmatism) was classified based on the type of refractive error in the keratoconic eye namely, emmetropia, myopia and hypermetropia (hyperopia). Emmetropia was defined as having dioptric power from -0.99 to 0.99 dioptres. Myopia was classified into low $(-1.00$ to $-2.99 \mathrm{D})$, moderate $(-3.00$ to -5.99$)$ and high $(-6.00 \mathrm{D}$ or worse). Regarding hypermetropia, mild $(+1.00$ to $+2.99 \mathrm{D})$ and moderate to high $(+3.00$ D or more extreme) was considered [17].

The data was entered and analyzed using Statistical Package for the Social Sciences (SPSS) version 25 software (SPSS Inc., Chicago, IL, USA). Shapiro-Wilk test was used to test for normality at $95 \%$ confidence interval (CI). Data on profile was analyzed descriptively and tabulated. Analysis of the effect of lens modalities (Corneal and scleral RGP) on the stages of keratoconus was performed using Kruskal Wallis test and all results presented in tables.

\section{Results.}

One hundred and twenty four patients' records were reviewed with mean age of $20.86 \pm 9.50$ (range; 9 - 67) years. Regarding gender distribution, 58.9\% were males while $41.1 \%$ were females. Keratoconic patients with contact lenses on both eyes (bilateral) were $57.3 \%$ while $26.6 \%$ and $16.1 \%$ had contact lenses worn in right and left eye respectively. Majority of contact lens wearers were of Afri- 
can origin (91.1\%) while minority was Asian with $8.9 \%$.

Nearly $55.6 \%$ did not have history on associated conditions although some (25\%) had signs of allergy. Contact lens intolerance was reported in $31.5 \%$ of the patients preceded by reduced vision with existing correction $(24.2 \%)$. In addition, the general visual blurred with halos accounted for $12.9 \%$, while change in prescriptions was made up of $5.6 \%$ of the total symptoms. Itching, ocular pain and reduced vision amounted to $8.9 \%$, while halos and squinting accounted for $12.9 \%$ of the total keratoconic patients. These results are illustrated in Table 1 below.

Table 1 showing demographic and presenting complaints of keratoconic contact lens.

There were 111 patients fitted with corneal RGP and only 13 patients fitted with scleral lenses whose visual acuity and refraction was assessed. The total eyes fitted with both corneal and scleral RGP contact lens modalities were 195. Sixty

Table 1. Summary of demographic profile of Keratoconic patient.

\begin{tabular}{|c|c|c|}
\hline Factors & $\mathrm{N}$ & Percentage (\%) \\
\hline \multicolumn{3}{|l|}{ Age } \\
\hline Mean & $20.85 \pm 9.503$ & \\
\hline Range & 9 to 67 & \\
\hline \multicolumn{3}{|l|}{ Gender } \\
\hline Male & 73 & 58.9 \\
\hline Female & 51 & 41.1 \\
\hline \multicolumn{3}{|l|}{ Laterality } \\
\hline Right eye & 33 & 26.6 \\
\hline Left eye & 20 & 16.1 \\
\hline Both Eyes & 71 & 57.3 \\
\hline \multicolumn{3}{|l|}{ Chief Complaints } \\
\hline Reduced Vision with habitual correction & 30 & 24.2 \\
\hline Contact lens intolerance & 39 & 31.5 \\
\hline General blurred vision with halos & 16 & 12.9 \\
\hline Change in habitual prescription & 7 & 5.6 \\
\hline Itching//pain//reduced vision & 11 & 8.9 \\
\hline Others & 21 & 16.9 \\
\hline \multicolumn{3}{|l|}{ Ethnicity } \\
\hline African & 113 & 91.1 \\
\hline Asian & 11 & 8.9 \\
\hline \multicolumn{3}{|l|}{ Associated condition } \\
\hline Ocular allergy & 24 & 19.4 \\
\hline Systemic diseases & 31 & 25.0 \\
\hline Not reported & 69 & 55.6 \\
\hline
\end{tabular}


Table 2. Visual acuity and refractive error change in Keratoconic stages.

\begin{tabular}{cccccc}
\hline & Mild & Moderate & Severe & H & p-value \\
\hline Corneal RGP (N) & 3 & 55 & 53 & & \\
Visual acuity & & & & 1.05 & 0.592 \\
Refractive change & & & & 1.62 & 0.443 \\
Scleral lenses (N) & 0 & 13 & 0 & & \\
Visual acuity & & & & 2.24 & 0.134 \\
Refractive change & & & & 1.14 & 0.285 \\
\hline
\end{tabular}

eight keratoconic patients (54.0\%) had moderate keratoconus, while fifty three patients $(43.5 \%)$ were severe cases and only three $(2.4 \%)$ had mild keratoconus. Most patients $(\mathrm{RGP}=55$, scleral $=13)$ who were fitted with lenses had moderate keratoconus while fifty three patients (fitted with RGP) had severe stage of keratoconus (none was fitted with scleral lenses). Furthermore, the study reported that three patients had mild keratoconus while the majority (67) had moderate and severe fifty three had severe keratoconus.

The distribution of refractive error among patients were; 122 high myopes, one low myopia and only one high hyperope. Visual acuity improved from $1.0 \pm$ $0.2 \log$ MAR to $0.00 \pm 0.3 \log$ MAR while refraction improved from $-12.00 \pm$ $01.02 \mathrm{D}$ to $-1.75 \pm 0.30 \mathrm{D}$ in the keratoconic eye fitted with contact lenses.

Analysis was performed on the effect of visual acuity and refractive error change, across the three severity stages. There were no significant differences in effects of lenses (RGP and Scleral lenses) across three stages in visual acuity and in three stages of severity $\mathrm{H}_{\mathrm{RGP}}(2)=1.05, p=0.59 ; \mathrm{H}_{\text {Scleral }}(1)=2.24, p=0.134$. Similar non-significant effect was observed in refractive error correction $\mathrm{H}_{\mathrm{RGP}}(2)$ $=1.62, p=0.44: \mathrm{H}_{\text {Scleral }}(1)=1.143, p=0.285$, as in Table 2 .

Table 2. Visual outcome in three stages of keratoconus based on keratometric grading.

\section{Discussion}

Keratoconic patients in the developing countries have limited access to quality contact lens care due to fewer practitioners and lack of sophisticated diagnostic equipment as well as variety of contact lens selection [18]. The study depicts the reality as most contact lenses wearers used corneal RGP lenses as compared to scleral contact lenses which could be due to lack of more options for selection. Most practitioners own slit lamps and keratometers to provide basic management while only few own corneal topographers. Furthermore, practitioners largely depended on patients complaints to make clinical decisions which may be prone to inaccurate or loss of information. The study was subjected to missing data in the records which could otherwise increase the sample size.

From the study on age, the keratoconic cases were young patients in their twenties suggesting productive age bracket in Kenya [19]. This study found a 
slightly higher average age than the results in Nepal [20] and lower than the Sudanese study [21]. The reason for this could be because patients seek an early intervention upon diagnosis in Kenya. Another study has shown that early intervention in $\mathrm{KC}$ management has demonstrated to be beneficial to most patients [22]. It is therefore clear in this study that patients might have benefitted in the first instance despite the lack of advanced equipment.

The sample size in this study showed a slightly higher number of males than females contact lens wearers. The findings are in agreement with the study by Chaudhary and Kandel [20] which found a higher number of males than females. However, the findings were contrary to the study by Chetty et al. [15] that cited more females than males. This could be because men are at a position to access better health care services unlike women due to cultural reasons [19]. Other studies have proposed that there is an equal preponderance in gender [23]. Therefore, there is no consensus on the most prevalent gender involvement.

Regarding laterality, $\mathrm{KC}$ has been shown to affect both eye depending on the time of onset and can only be detected based on definitions and detection criteria [4]. In this study, KC might have affected the patients earlier than expected, however detection and correction may have delayed. This study presents contact lens wearers rather than general profile of KC patients.

Most of the patients had contact lenses worn on both eyes. The ability to wear lens could have been based on the severity of KC [1] as well as ability to purchase the contact lenses.

Most patients were of African origin because Kenya is largely inhabited by Africans and fewer Asians. Majority of the patients were referred from the countryside which may have contributed to a higher number of Africans in the study.

Patients' complaints are key to diagnosis as witnessed in the study [24]. However, the majority of the patients did not report history of associated conditions leading to the conclusion of insufficient collaboration of multidisciplinary approach in patients' management.

Allergic signs which involved conjunctival and lids signs were observed and recorded. This implied that some patients had allergic conditions even though they did not report. Allergies as associated conditions are more common in tropical regions which may explain the reason for increased prevalence in this study because it was conducted in Sub-Saharan Africa (considered to be tropical region). Eye rubbing is a common phenomenon that creates corneal distortion and results in development of keratoconus over time which is thought to affect patients living in tropical regions [25].

Contact lens intolerance and reduced vision with habitual correction were also reported. Intolerance results from contact lens complications as well as contact lens inherent properties such as material properties [26]. In this study, the intolerance rate suggested the need to concentrate on compliance as well as care. The change of prescription suggested the progression of keratoconus and the rate of 
increased refractive error. Keratoconus progresses with increasing of refraction which provides for the change in contact lens prescription. As the prescription changes, patients find it difficult to wear the present prescription which gives rise to haloes and the subsequent reduced vision.

This study noted an improved identification of keratoconus by practitioners as compared to the previous trend because there were reduced severe cases [12]. Moreover, the findings could postulate that there is an increased awareness among practitioners in Kenya [5]. Since the study focused on corneal RGP and sclerals lens wearers, it was limited by a small sample size and inadequate scleral lenses. The present study indicated that there was no significant difference in visual acuity and refraction of patients at different $\mathrm{KC}$ stages. However, there was no patient fitted with scleral lens at mild and severe stages. This could be because such patients opted for corneal transplants as an option with lack of a better contact lens option or inadequate information. It is therefore important to have all patients informed and scleral lenses tried while considering corneal surgery as the last option at an advanced stage of keratoconus.

This study demonstrated that given equal visual acuity and refractive correction (Table 2), patients' subjective factors such as comfort and ease of lens handling could be beneficial to the patients for successful management. The study agrees with other findings elsewhere in the Nepalese study and Ghanaian population [13] [20]. This would explain why most practitioners in Africa prefer rigid gas permeable lenses in keratoconic fitting in addition to affordability and other factors [1] [14]. The study was limited by the small number of scleral contact lens wearers which necessitated the use of non-parametric data analysis. Furthermore, the secondary nature of data limited the design since as experimental design would provide more information.

\section{Conclusion}

The study demonstrated the ability of keratoconic contact lenses to improve visual acuity and reduce refraction at different keratometric graded severity. Despite limited resources, the available grading based present resources could improve the patients' outcome. Given better visual outcome, factors such as patients comfort, affordability and care modalities could be considered while making decisions to prescribe the lens modalities.

\section{Conflicts of Interest}

The authors declare no conflicts of interest regarding the publication of this paper.

\section{References}

[1] Downie, L.E. and Lindsay, R.G. (2015) Contact Lens Management of Keratoconus. Clinical and Experimental Optometry, 98, 299-311.

https://doi.org/10.1111/cxo.12300 
[2] Hassani, M., Jafarzadehpur, E., Mirzajani, A., Yekta, A. and Khabazkhoob, M. (2018) A Comparison of the Visual Acuity Outcome between Clearkone and RGP Lenses. Journal of Current Ophthalmology, 30, 85-86. https://doi.org/10.1016/j.joco.2017.08.006

[3] Mohammadpour, M., Heidari, Z. and Hashemi, H. (2018) Updates on Managements for Keratoconus. Journal of Current Ophthalmology, 30, 110-124. https://doi.org/10.1016/j.joco.2017.11.002

[4] Gokhale, N.S. (2013) Epidemiology of Keratoconus. Indian Journal of Ophthalmology, 61, 382-383. https://doi.org/10.4103/0301-4738.116054

[5] Amanzadeh, K., Karimian, F. and Khanlari, M. (2012) Management of Keratoconus. Journal of Ophthalmic \& Vision Research, 7, 341-346.

[6] Mahadevan, R., Arumugam, A.O., Arunachalam, V. and Kumaresan, B. (2009) Keratoconus-A Review from a Tertiary Eye-Care Center. Journal of Optometry, 2, 166-172. https://doi.org/10.3921/joptom.2009.166

[7] Wu, Y., Tan, Q., Zhang, W., Wang, J., Yang, B., Ma, W., et al. (2015) Rigid Gas-Permeable Contact Lens Related Life Quality in Keratoconic Patients with Different Grades of Severity. Clinical and Experimental Optometry, 98, 150-154. https://doi.org/10.1111/cxo.12237

[8] de Luis Eguileor, B., Etxebarria Ecenarro, J., Santamaria Carro, A. and Feijoo Lera, R. (2018) Irregular Corneas: Improve Visual Function With Scleral Contact Lenses. Eye Contact Lens, 44, 159-163. https://doi.org/10.1097/ICL.0000000000000340

[9] Koppen, C., Kreps, E.O., Anthonissen, L., Van Hoey, M., Dhubhghaill, S.N. and Vermeulen, L. (2018) Scleral Lenses Reduce the Need for Corneal Transplants in Severe Keratoconus. American Journal of Ophthalmology, 185, 43-47. https://doi.org/10.1016/j.ajo.2017.10.022

[10] Masiwa, L.E. and Moodley, V. (2020) A Review of Corneal Imaging Methods for the Early Diagnosis of Pre-Clinical Keratoconus. Journal of Optometry. https://doi.org/10.1016/j.optom.2019.11.001

[11] Al-Mahrouqi, H.H., Al-Shamli, N., Mohan, N.R., Oraba, S.B., Panchatcharam, S.M., Al-Saidi, R., et al. (2018) Clinical Profile of Omani Keratoconus Patients: An Experience from a Tertiary Referral Centre in Muscat. Oman Journal of Ophthalmology, 11, 259-264. https://doi.org/10.4103/ojo.OJO $203 \quad 2017$

[12] Rashid, Z.A., Millodot, M. and Evans, K.S.E. (2016) Characteristics of Keratoconic Patients Attending a Specialist Contact Lens Clinic in Kenya. Middle East African Journal of Ophthalmology, 23, 283-287. https://doi.org/10.4103/0974-9233.194074

[13] Nelson-Ayifah, D., et al. (2017) Profile of Keratoconus in Selected Eye Clinics, Accra: A Retrospective Study. Ophthalmology and Vision Science, 1, 8-16.

[14] Munsamy, A.J., Moodley, V.R., Naidoo, P., Mangwarara, T.R., Abdullah, R., Govender, D., et al. (2015) A Frequency Analysis of Cone Characteristics for the Different Stages of Keratoconus. African Vision and Eye Health, 74, a302. https://doi.org/10.4102/aveh.v74i1.302

[15] Chetty, E. and Rubin, A. (2019) Preliminary Demographics for Patients with Keratoconus Attending a University-Based Clinic in Johannesburg, South Africa. African Vision and Eye Health, 78, a472. https://doi.org/10.4102/aveh.v78i1.472

[16] Zadnik, K., Barr, J.T., Edrington, T.B., Everett, D.F., Jameson, M., McMahon, T.T., et al. (1998) Baseline Findings in the Collaborative Longitudinal Evaluation of Keratoconus (CLEK) Study. Investigative Ophthalmology \& Visual Science, 39, 2537-2546.

[17] Cumberland, P.M., Chianca, A. and Rahi, J.S. (2016) Accuracy and Utility of 
Self-Report of Refractive Error. JAMA Ophthalmology, 134, 794-801. https://doi.org/10.1001/jamaophthalmol.2016.1275

[18] Ezinne, N.E., Austin, E., Ilechie, A.A. and Mashige, K.P. (2019) Contact Lens Prescribing Patterns in Abuja, Nigeria. Journal of the Nigerian Optometric Association, 21, 26-32.

[19] Kenya National Bureau of Statistics (KNBS) (2013) Exploring Inequality. Nairobi.

[20] Chaudhary, M. and Kandel, H. (2017) Visual Outcome in Keratoconus with Spherical Rigid Gas Permeable Contact Lens. Journal of the Institute of Medicine, 39, 8-11.

[21] Abdu, M., Mohd Ali, B. and Binnawi, K. (2015) Corrected Visual Functions in Keratoconus Patients with Rigid Gas-Permeable Contact Lens and Its Association with Cone Location. Sudanese Journal of Ophthalmology, 7, 48-52. https://doi.org/10.4103/1858-540X.169436

[22] Shi, Y. (2016) Strategies for Improving the Early Diagnosis of Keratoconus. Clinical Optometry, 8, 13-21. https://doi.org/10.2147/OPTO.S63486

[23] Althomali, T.A. (2018) Relative Proportion of Different Types of Refractive Errors in Subjects Seeking Laser Vision Correction. The Open Ophthalmology Journal, 12, 53-62. https://doi.org/10.2174/1874364101812010053

[24] Balcer, L.J., Miller, D.H., Reingold, S.C. and Cohen, J.A. (2015) Vision and Vision-Related Outcome Measures in Multiple Sclerosis. Brain, 138, 11-27. https://doi.org/10.1093/brain/awu335

[25] Roy, S., Yadav, S., Dasgupta, T., Chawla, S., Tandon, R. and Ghosh, S. (2019) Interplay between Hereditary and Environmental Factors to Establish an in Vitro Disease Model of Keratoconus. Drug Discovery Today, 24, 403-416. https://doi.org/10.1016/j.drudis.2018.10.017

[26] Bhamra, T.S. and Tighe, B.J. (2017) Mechanical Properties of Contact Lenses: The Contribution of Measurement Techniques and Clinical Feedback to 50 Years of Materials Development. Contact Lens Anterior Eye, 40, 70-81.

https://doi.org/10.1016/j.clae.2016.11.005 